\title{
1 Localization of the putative recombinase Pf-int to the apicoplast of
}

\section{Plasmodium falciparum}

3

4 A. V. Berglar ${ }^{1,2, *}$, S. S. Vembar ${ }^{3, * * *}$, D. N. Gopaul ${ }^{1, * *}$

5

6 'Department of Genomes and Genetics, Unité Plasticité du Génome Bactérien, CNRS, UMR 3525, Institut Pasteur,

7 Université de Paris, F75015, Paris, France

$8 \quad{ }^{2}$ FdV (now : FIRE) Doctoral School 474 Centre de Recherches Interdisciplinaires, Université de Paris, Paris, France

$9 \quad{ }^{3}$ Département de Parasites et Insectes Vecteurs, Unité Biologie des Interactions Hôte-Parasite, Institut Pasteur,

10 Université de Paris, CNRS, ERL 9195, INSERM, UMR 1201, F-75015 Paris, France

$11{ }^{*}$ Current affiliation : SciVisLab - Scientific Visualisation Lab, Cologne, Germany

$12{ }^{* *}$ Current affiliation : Department of Education, Institut Pasteur, Université de Paris, F-75015 Paris, France

$13{ }^{* * *}$ Current affiliation : Institute of Bioinformatics and Applied Biotechnology, Bengaluru, India

14

15

16 Corresponding author

17 E-mail: deshmukh.gopaul@pasteur.fr (DNG) 


\section{Abstract}

19 Diseases caused by apicomplexan parasites, such as malaria and toxoplasmosis cause $\sim 200$ million

20 (worldwide) and 1 million (Europe) infections, respectively, every year. Apicomplexa possess a

21 non-photosynthetic organelle homologous to the plant chloroplast, the so-called apicoplast, that is

22 essential for their growth and survival. This study focused on the Int recombinase, the first protein

23 discovered in Plasmodium spp. with the features of a site-specific recombinase, and which has an

24 apicoplast targeting leader sequence at its amino-terminus. Int is conserved amongst several apicomplexan parasites. In the human toxoplasmosis parasite, Toxoplasma, Int localizes to the apicoplast and Pf-Int, the P. falciparum member, belongs to the group of non-mutable essential genes in $P$. falciparum. A conserved protein that has been shown to be essential at least in one species and that localizes to an essential organelle may become a novel drug target. Therefore, the aim of this study was to confirm the sub-cellular localization of Int in the human malaria parasite $P$. asexual blood stages, we observed that Int partially co-localized with the apicoplast (to discrete foci adjacent to the nucleus). 
Parasites of the phylum apicomplexa cause diseases like malaria and toxoplasmosis and are therefore an important health and socio-economic threat to mankind. The eukaryotic parasite Plasmodium falciparum is the main causative agent of Malaria, which generates $\sim 200$ million infections and $\sim 400,000$ deaths every year [1]. Toxoplasma gives rise to toxoplasmosis, a severe disabling condition, which is responsible for over one million infections per year in the European region through contaminated food [2]. The lack of effective vaccines highlights the need for novel drug targets against these organisms. membranes and is homologous to the plant chloroplast. It has been hypothesized to have been evolutionarily derived by secondary endosymbiosis. Even though this plastid has lost the ability to perform photosynthesis over time, it has been retained and serves essential purposes such as fatty acid, isoprenoid precursor, Fe-S cluster and heme biosyntheses[5-7]. The apicoplast carries 1 to 15 copies of a mostly circular $35 \mathrm{~kb}$ genome and autonomously performs replication synchronized with schizogony, transcription and translation events [8-10]. These events require plastid DNA copy number management. None of the proteins expressed by the apicoplast genome fulfills this role. The majority of all apicoplast proteins, however, are nuclear-encoded and are targeted to the apicoplast via a N-terminal bipartite targeting sequence, consisting of a translocation sequence and a transit peptide [11-13] (Fig 1). Once imported to the apicoplast, these nuclear-encoded proteins complement the 30 apicoplast-encoded proteins for all remaining plastid activities [14-16].

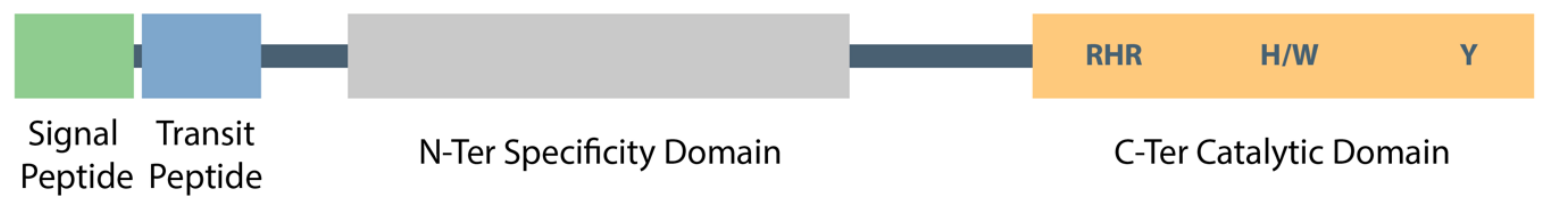


Fig 1. Int protein domain map. Ints as members of Tyr-recombinases are composed of multiple domains. In apicomplexans they also carry a targeting peptide in their N-terminus. The main Nterminal region in Tyr-recombinases are variable in sequence, providing specificity. The C-terminal catalytic domain carries the conserved active site residues involved in DNA cleavage, strand exchange and religation. All members of the Apicomplexa shown here carry the conserved residues of the catalytic domain: R-K/H-H-R-H/W-Y (S1 Fig).

We previously proposed the nuclear-encoded protein Pf-Int (PF3D7_1308800) of Plasmodium falciparum to be an integrase and we partially characterized it as a putative tyrosine site-specific recombinase (Y-SSR) for DNA binding [17]. Pf-Int has been shown to be essential in a study by Zhang et al. (2018) [18]. It was part of the $2.9 \%$ of genes that were not only non-mutable, but that also did not have any Piggy Bac transposon insertions even in their intergenic regions. SSRs in general are involved in a variety of cellular processes such as genome replication, recombination and repair, the impairment of which results in malfunctioning of the organism or interference in the mobility of genetic elements [19-22]. The SSRs require a DNA recombination site ranging between 30 to 200 base pairs in length. These sites contain two motifs with partial inverted repeat (IR) symmetry that flank a central crossover region where DNA strand exchange occurs [23]. Potential outcomes of a recombination event can be insertion, excision or inversion [19] depending on the orientation of the two sites. In practice, this mechanism is used for genome decatenation, partitioning or gene shuffling.

Pf-Int is composed of 490 amino acids $(\sim 58 \mathrm{kDa})$ and is conserved among six members of Plasmodium spp., namely the human infecting P. falciparum, P. vivax and P. knowlesi, as well as the rodent infecting $P$. berghei, $P$. yoelii and P. chabaudi. Conservation of the C-terminal core part of the protein extends to other members of Apicomplexa in the branches Hematozoa and Coccidia, which are all obligate parasites. Using BLAST, we were able to identify the un-annotated homologs 
of Pf-Int in Vitrella brassicaformis and Eimeria maxima (S1 Fig and S2 Fig). This points to the universal distribution of Int among Apicomplaxa that have retained an apicoplast during evolution.

Pf-Int shares homology with other well-known Y-SSRs, such as phage $\lambda$-Int, P1 phage Cre, bacterial XerC/XerD, the integron encoded VchInt1b (Int4) of Vibrio cholerae, and P. aeruginosa Int1 mainly in terms of active site residues and polypeptide length of the catalytic domain (amino acid 192-490) [24]. Pf-Int and its homologs in apicomplexa are predicted to encode substantial Nterminal extensions thought to act as plastid-targeting peptides [11]. Nuclear-encoded apicoplast proteins that are targeted into the organelle require multiple trafficking steps from the outermost membrane through the subsequent intermembrane compartments into the lumen [25] (Fig 2).

Typically, the upstream portion of the bipartite leader acts as a classical signal peptide that facilitates the co-translational insertion of the protein into the rough endoplasmic reticulum (ER). After cleavage of the signal peptide (SP) by the signal peptidase, the downstream N-terminal transit peptide, similar to those found in plants, is exposed. This transit peptide (TP) directs the trafficking of the protein into the stroma of the apicoplast over either of two different pathways, directly or through the golgi (for details see Fig 2). 


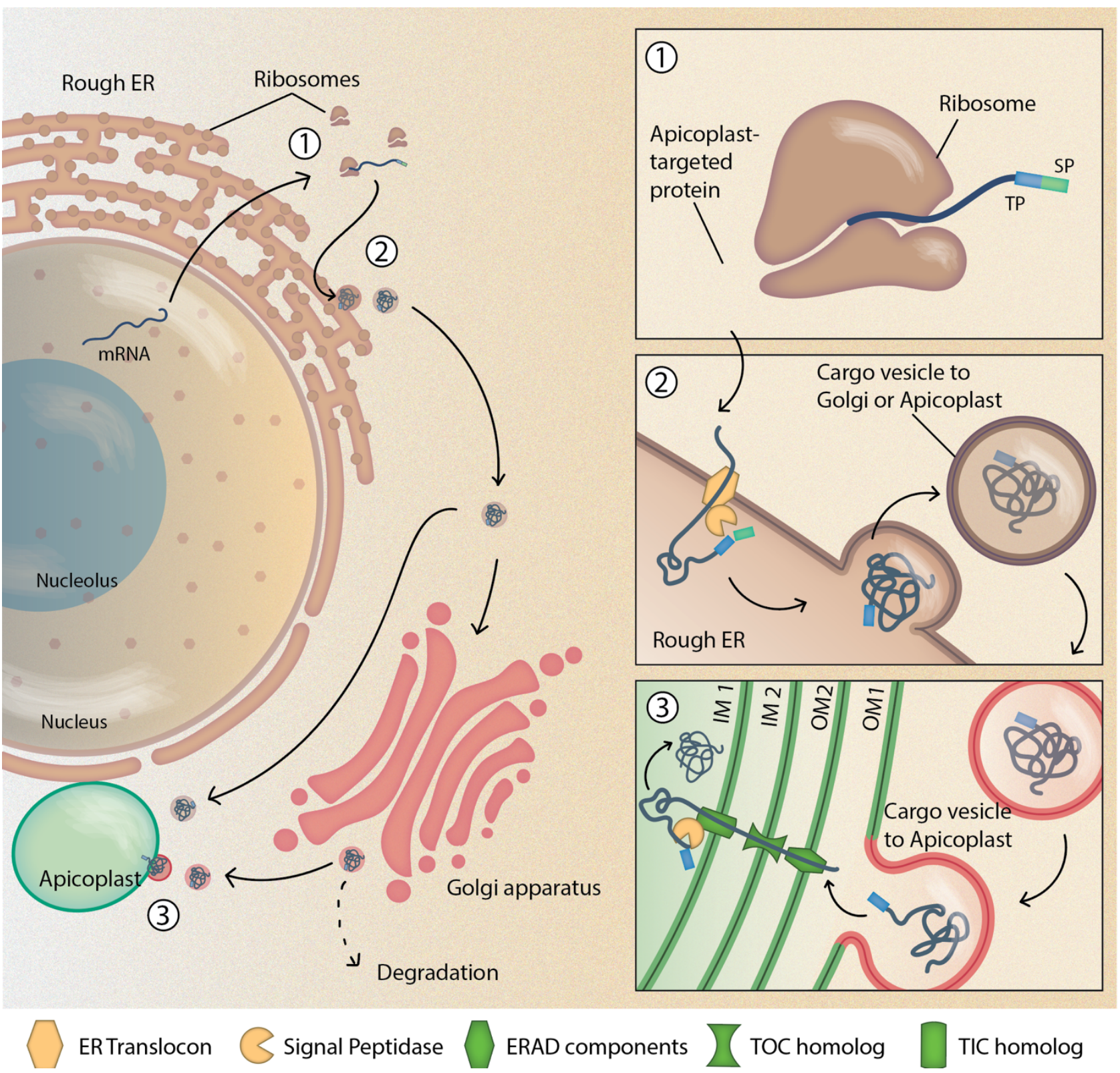

Fig 2. Protein targeting to the apicoplast. 1. The apicoplast destined protein is translated at the ribosome. It has a bipartite leader sequence consisting of a signal peptide (SP, green), which directs the protein to the ER, and a transit peptide (TP, blue), which later mediates the transport across the apicoplast membranes. 2. From the endoplasmic reticulum (ER), where the SP is cleaved off by a peptidase, ER-vesicles carry the cargo either directly to the apicoplast (11), or to the Golgi apparatus, a branchpoint for protein sorting $(23,24)$. 3. Golgi vesicles then fuse with the outermost membrane (OM1) of the apicoplast. Subsequently, the TP guides it across the second outermost membrane (OM2) via an ER-associated degradation (ERAD)-like translocon (25). The second 
112 innermost membrane (IM2) is crossed via the outer chloroplast-like membrane (TOC) and the

113 innermost membrane (IM1) via the inner chloroplast-like membrane (TIC) translocons,

114 respectively. In the apicoplast stroma, the TP is cleaved off and the protein adopts its final

115 conformation. Proteins with transmembrane domains anchor themselves in one of the membranes

116 (26,27). Figure C AVB.

117

118

119 residues and protein domains associated with integrases. We hypothesize the role of Int in the

120 apicoplast to be associated with plastid DNA. The homologous protein of Pf-Int in Toxoplasma

121 gondii (Tg-Int; TGME49_259230, previously TGME49_059230) had independently been identified

122 during a screen of novel apicoplast-resident proteins [26]. The coding sequence of Tg-Int includes a

123 phage integrase domain and a SAP motif. SAP domain proteins are very well conserved from yeast

124 to human, and have been shown to be involved in DNA repair [27], as well as in chromosomal

125 organization [28]. Here, we provide evidence for the co-localization of the integrase to the

126 apicoplast in P.falciparum. 


\section{Antibodies used in this study}

Table 1 Antibodies used in this study.

\begin{tabular}{|c|c|c|c|c|c|c|}
\hline & \multicolumn{3}{|c|}{ Primary antibodies } & \multicolumn{3}{|c|}{ Secondary antibodies } \\
\hline Name & $\begin{array}{l}\text { Rat Anti Pf- } \\
\text { Int C192 }\end{array}$ & $\begin{array}{l}\text { Rat Anti Pf- } \\
\text { Int C192 }\end{array}$ & $\begin{array}{l}\text { Anti-HA } \\
\text { tag } \\
\text { antibody } \\
{[12 \mathrm{CA} 5]} \\
(\text { ab1424) }\end{array}$ & $\begin{array}{l}\text { Amersham } \\
\text { ECL Rat } \\
\text { IgG, HRP- } \\
\text { linked } \\
\text { whole } \\
\text { antibody } \\
\text { (from goat) }\end{array}$ & $\begin{array}{l}\text { Goat anti-Rat } \\
\text { IgG }(\mathrm{H}+\mathrm{L}) \\
\text { Cross- } \\
\text { Adsorbed } \\
\text { Secondary } \\
\text { Antibody, } \\
\text { Alexa Fluor } \\
488\end{array}$ & $\begin{array}{l}\text { Goat anti- } \\
\text { Mouse IgG } \\
(\mathrm{H}+\mathrm{L}) \text { Cross- } \\
\text { Adsorbed } \\
\text { Secondary } \\
\text { Antibody, } \\
\text { Alexa Fluor } \\
568\end{array}$ \\
\hline Clonality & Polyclonal & Polyclonal & Monoclonal & $\mathrm{n} / \mathrm{a}$ & Polyclonal & Polyclonal \\
\hline Host species & Rat & Rat & Mouse & Goat & Goat & Goat \\
\hline $\begin{array}{l}\text { Commercial supplier } \\
\text { or source laboratory }\end{array}$ & Eurogentech & Eurogentech & AbCam & $\begin{array}{l}\text { Amersham } \\
\text { (GE } \\
\text { Healthcare) }\end{array}$ & $\begin{array}{l}\text { ThermoFisher } \\
\text { Scientific }\end{array}$ & $\begin{array}{l}\text { ThermoFisher } \\
\text { Scientific }\end{array}$ \\
\hline $\begin{array}{l}\text { Catalogue or clone } \\
\text { number }\end{array}$ & $\begin{array}{l}\text { n/a } \\
\text { custom- } \\
\text { synthesized }\end{array}$ & $\begin{array}{l}\text { n/a } \\
\text { custom- } \\
\text { synthesized }\end{array}$ & ab1424 & NA935 & A-11006 & A-11004 \\
\hline Batch number & $\mathrm{n} / \mathrm{a}$ & $\mathrm{n} / \mathrm{a}$ & $\mathrm{n} / \mathrm{a}$ & $\mathrm{n} / \mathrm{a}$ & $\mathrm{n} / \mathrm{a}$ & $\mathrm{n} / \mathrm{a}$ \\
\hline $\begin{array}{l}\text { Antigen(s) used to } \\
\text { raise the antibody }\end{array}$ & $\begin{array}{l}\text { Purified } \\
\text { recombinant } \\
\text { Pf-Int C192 }\end{array}$ & $\begin{array}{l}\text { Purified } \\
\text { recombinant } \\
\text { Pf-Int C192 } \\
\end{array}$ & $\mathrm{n} / \mathrm{a}$ & $\mathrm{n} / \mathrm{a}$ & $\mathrm{n} / \mathrm{a}$ & $\mathrm{n} / \mathrm{a}$ \\
\hline $\begin{array}{l}\text { Public identifier from } \\
\text { the Antibody Registry }\end{array}$ & $\mathrm{n} / \mathrm{a}$ & $\mathrm{n} / \mathrm{a}$ & AB_301017 & AB_772207 & AB_2534074 & AB_2534072 \\
\hline $\begin{array}{l}\text { Final antibody } \\
\text { concentration or } \\
\text { dilution }\end{array}$ & $1: 500(\mathrm{WB})$ & $1: 250$ (IFA) & $1: 500$ (IFA) & $\begin{array}{l}1: 10,000 \\
(\mathrm{WB})\end{array}$ & $1: 1000$ (IFA) & $1: 1000$ (IFA) \\
\hline See figure & $3 \mathrm{~A}$ & $3 \mathrm{~B}, 3 \mathrm{C}$ & $3 \mathrm{C}$ & $3 \mathrm{~A}$ & $3 \mathrm{~B}, 3 \mathrm{C}$ & $3 \mathrm{C}$ \\
\hline
\end{tabular}

\section{P. falciparum parasite culture}

133 Red blood cells were obtained from the Etablissement Français du Sang of Necker hospital, Paris,

134 under agreement with Institut Pasteur, and following the guidelines for informed consent of donors

135 for the use of blood or its derivatives for research purposes. The red blood cells were completely

136 de-identified before researchers accessed the samples. P. falciparum blood stage parasites from the

137 3D7 strain [29] were cultured using modifications to the method described by Trager and Jensen

138 [30]. Parasites were grown in $0^{+}$human erythrocytes in RPMI 1640 medium containing l-glutamine

139 (Invitrogen) supplemented with 2X Hypoxanthine, $50 \mu \mathrm{g} / \mathrm{ml}$ gentamycine, $5 \%(\mathrm{v} / \mathrm{v})$ human serum

140 (PAA Laboratories $\mathrm{GmbH}$ ) and $5 \% \mathrm{v} / \mathrm{v}$ Albumax II (Invitrogen) at $37^{\circ} \mathrm{C}$ in a gas environment of 5 
$141 \% \mathrm{CO}_{2}, 5 \% \mathrm{O}_{2}$ and $90 \% \mathrm{~N}_{2}$ in Falcon culture flasks. The culture medium was changed every $48 \mathrm{~h}$

142 by aspiration and the parasite were diluted in fresh RBCs according to parasitemia. Synchronization 143 of cultures consisted of two consecutive 5\% sorbitol (Sigma) treatments [31]. We estimated by 144 giemsa staining that the parasites were synchronized within a window of $\sim 6 \mathrm{~h}$.

\section{Fractionation of $P$. falciparum total protein content}

147 The total protein content of synchronized $P$. falciparum parasites was fractionated into cytoplasmic 148 and nuclear fractions after Oehring et al. (2012) [32] with some modifications. Briefly, parasites $1492 \times 10^{10}$ ring stages (2-20 hpi); $10^{10}$ trophozoites (20-34 hpi); $5 \times 10^{9}$ schizonts $(34-48$ hpi) were

150 released from RBCs by saponin lysis and washed three times in PBS. Parasites were lysed in a 151 hypotonic cytoplasmic lysis buffer CLB (20 mM HEPES (pH 7.9), 10 mM KCl, 1 mM EDTA, 1 152 mM EGTA, $0.65 \%$ NP-40, 1 mM DTT, Complete TM protease inhibitors (Roche Diagnostics)) for $1535 \mathrm{~min}$ on ice. Nuclei were pelleted at $2,000 \mathrm{x} \mathrm{g}$ for $5 \mathrm{~min}$ and the supernatant saved (cytoplasmic extract; fraction 1). After four to seven washes in CLB the pellet was solubilized in $1.5 \mathrm{ml}(3 \mathrm{ml})$ SDS extraction buffer SEB (2 \% SDS, 10 mM Tris-HCl (pH 7.5)) for 20 min under constant agitation at room temperature, cleared by centrifugation at 13,000 rpm for $15 \mathrm{~min}$ and saved (SDS extract; fraction 2). Large volumes for SDS-PAGE were concentrated by trichloracetic acid (TCA) precipitation.

\section{TCA precipitation}

161 One volume of TCA stock $(100 \%(\mathrm{w} / \mathrm{v}) \mathrm{TCA})$ was added to four volumes of protein sample and

162 incubated for $10 \mathrm{~min}$ at $4^{\circ} \mathrm{C}$. The samples were spun in a microcentrifuge at $14,000 \mathrm{rpm}$ for $5 \mathrm{~min}$.

163 The protein pellet was washed twice with $200 \mu$ cold acetone (Carlo Erba Reagents). The pellet 164 was dried by placing the tube in a $95^{\circ} \mathrm{C}$ heat block for 5-10 min. For SDS-PAGE, sample buffer was added and the samples were heated for $10 \mathrm{~min}$ at $95^{\circ} \mathrm{C}$ before loading onto the gel. 


\section{Western blot analysis of Pf-int expression in blood stage parasites}

An SDS-PAGE with fractionated protein samples was run in 6x SDS Loading buffer (Laemmli, 2\%

SDS) to a final concentration of 1x. Samples were run on a 4-12\% Bis-Tris SDS gel (NuPage,

Invitrogen), $40 \mathrm{~min}$ at $200 \mathrm{~V}$ in MES running buffer (Novex ${ }^{\circledR}$ Invitrogen) or $60 \mathrm{~min}$ at $180 \mathrm{~V}$ in

MOPS (Novex® Invitrogen) running buffer. As molecular marker, we used the PAGE RulerTM

Prestained Protein ladder 10-180 kDa (Fermentas). Recombinant and purified Pf-int was used as a control. Its preparation is described elsewhere [17].

Western blot analyses were carried out using a rat antibody raised against purified recombinant Pfint (aa 192-490) (Eurogentec, Belgium). The protein content was transferred to a nitrocellulose membrane using the Trans Blot Turbo (BioRad) device program MIXED MW (5-150 kDa, 7 min, 1.3 A up to $25 \mathrm{~V}$ ). The membrane was immediately placed into saturation buffer and incubated for 1 hour at RT with agitation, then incubated with the primary antibody at 1:500 dilution for $1 \mathrm{~h}$ at $\mathrm{RT}$ or $\mathrm{o} / \mathrm{n}$ at $4^{\circ} \mathrm{C}$. The membrane was then incubated with the secondary antibody (1:1000 dilution of goat arat-HRP) for $1 \mathrm{~h}$ at $\mathrm{RT}$ or o/n at $4{ }^{\circ} \mathrm{C} .4 \mathrm{x} 5 \mathrm{~min}$ Washing buffer washes at $\mathrm{RT}$ and with agitation were done after each incubation step. Blots were developed using the femto kit (Thermo Fisher Scientific).

\section{Localization studies in $P$. falciparum via Immunofluorescence assay}

Localization studies were carried out using a rat antibody raised against purified recombinant Pf-int min at RT with $15 \mu 1 \mathrm{PBS}+1 \%$ BSA (filter sterilized, Sigma Aldrich). The primary antibody

190 solution was prepared in PBS $+1 \%$ BSA. The optimal antibody concentration was determined by

191 dilution series. No primary antibody or pre-immune serum controls were used. $10 \mu 1$ of primary 
193 at RT or o/n at $4^{\circ} \mathrm{C}$. Each well was washed thrice with PBS for 5 min at RT. $10 \mu$ l of the secondary

194 antibody (AlexaFluor $\left.{ }^{\circledR}\right)$, prepared in PBS $+1 \%$ BSA, were added to each well at a dilution of

195 1:1000 and incubated for 30 min at RT. Each well was washed thrice with PBS $+0.5 \%$ Tween-20

196 for $5 \mathrm{~min}$ at RT. After the final wash, $3 \mu \mathrm{l}$ of PROLONG Gold Antifade + DAPI solution were

197 added to each well and the slide was covered with a clean coverslip (Menzel) and sealed with nail

198 varnish. The finished slide was visualized under an Eclipse 80i microscope (Nikon).

199 


\section{Results}

In this study, we performed immunolocalization studies of a putative apicomplexan integrase Int using immunofluorescence assays (IFA) in P. falciparum. In the following, we report the results of our investigation.

\section{P. falciparum Int is translocated to the apicoplast}

To determine the localization of Pf-Int in asexual blood stages of $P$. falciparum, cellular fractionation studies and western blot analysis were performed with rat anti-Pf-Int antibodies on $P$. falciparum cellular extracts. In general, the cytoplasmic fraction contains soluble proteins while the nuclear fraction contains nuclear, nuclear-associated, membrane-associated as well as intraorganellar, therefore also apicoplast proteins [32]. Fig 3A (top row lane 3) shows the characterization of Pf-Int in whole cell extract (wce), cytoplasmic (cyt) and nuclear (nuc) fractions for wild-type $P$. falciparum 3D7 ring stage parasites. The control shows a purified $35 \mathrm{kDa}$ recombinant fragment of Pf-Int (C192) reaching from amino acid 192 to 490, the C-terminus [17]. A band of $\sim 60 \mathrm{kDa}$ in size corresponding to the full length Pf-Int was identified in the nuclear fraction, therefore potentially associated with the apicoplast.

Additionally, immunolocalization studies were performed using anti-Pf-Int antibodies on a mixed population of ring, trophozoite and schizont stage parasites of the wild-type 3D7 strain. Pf-Int was localized to a region adjacent to the nucleus, predominantly as a single punctum (Fig 3B, columns 2 and 3). This finding was in accordance with our results from the cellular fractionation studies.

Based on these results, the co-localization of Pf-Int was investigated with the apicoplast marker outer-apicoplast-membrane triose phosphate transporter (PfoTPT) in TPT-HA transformed parasites [33]. The co-localization of Pf-Int with the apicoplast marker was confirmed (Fig 3C, columns 5, 7 and 8). 
A

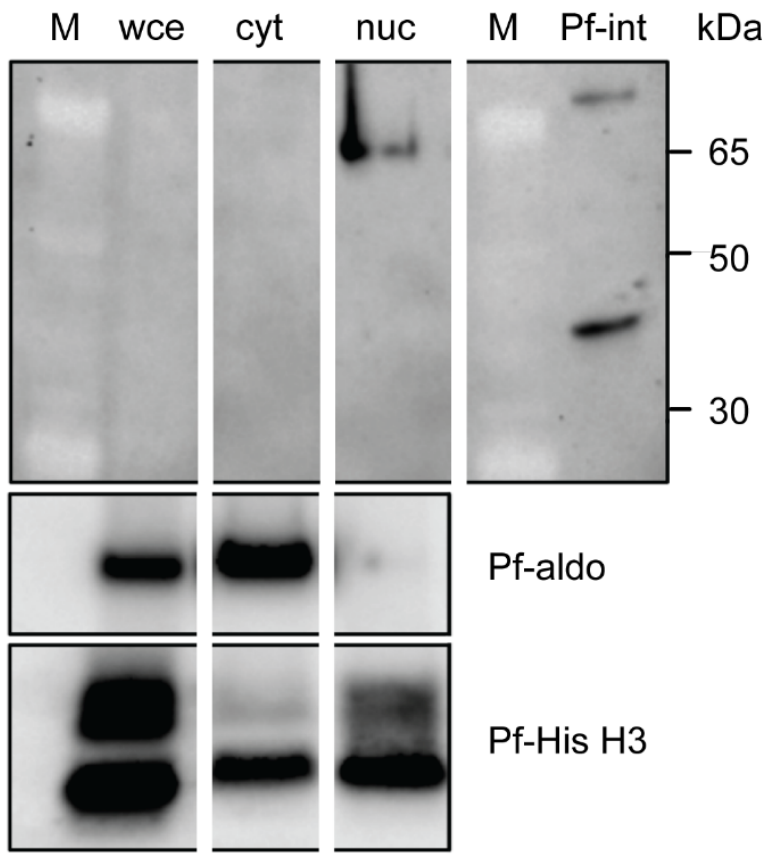

B

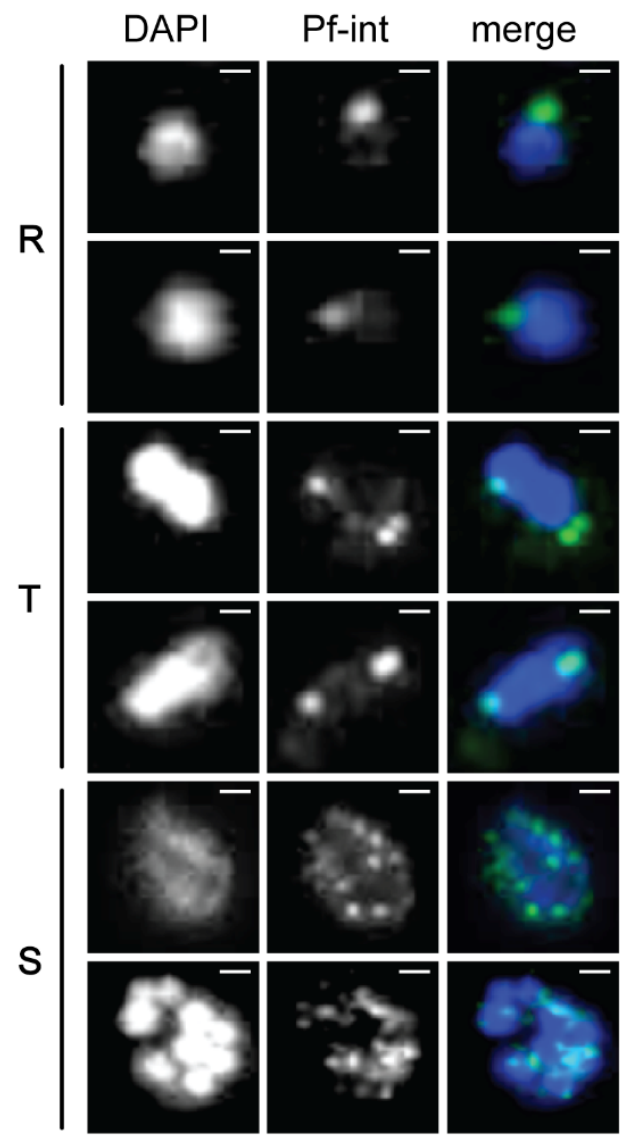

C

227 Fig 3. Subcellular localization of Pf-int. A) Pf-int localization studies by western blot. Detection

228 of Pf-Int in synchronized P. falciparum 3D7 parasites: Ring stage whole cell extract (wce),

229 cytoplasmic (cyt) and nuclear (nuc) fractions. rat-Anti-Pf-Int primary antibody dilution 1:500,

230 secondary goat-anti-rat-HRP dilution 1:10,000. Developed with femto kit (ThermoFisher

231 Scientific). Positive control: purified recombinant Pf-Int (C192, 35 kDa, $0.1 \mu \mathrm{g})($ Top Row panel 4).

232 Loading/ fractionation controls with cytoplasmic marker Pf-aldolase (Pf-aldo) and nuclear marker

233 Pf-Histone H3 (Pf-His H3)(Lower panel). B) Initial characterization of anti-Pf-Int in

234 immunolocalization studies. Anti-Pf-Int gave a punctate pattern, partly at the nuclear periphery 
235 (Panel 2 and 3). Shown for 3D7 ring (R), trophozoite (T) and schizont (S) stages. Anti-Pf-Int 236 (1:250), secondary antibody AlexaFluor®488 (1:1000). The nuclei were stained with DAPI (Panel 237 1). Images are shown in duplicate for all parasite stages (Black vertical bar). C) Left panels: Anti238 hemagglutinin (HA, green)-tagged PfoTPT localizes adjacent to the nucleus (blue) in PfoTPT-HA 239 parasites. Right panel: Anti-Pf-Int (green) co-localizes with apicoplast-located PfoTPT (red) in 240 PfoTPT-HA parasites. Each study is shown for ring (R) stages. Anti-Pf-Int primary antibody 241 (1:250), mouse-anti-HA primary antibody (1:500); secondary antibodies AlexaFluor ${ }^{2} 488$ (green) 242 or AlexaFluor ${ }^{\circledR 568}$ (red) (1:1000). The nuclei were stained with DAPI. Images are shown in 243 duplicate. Scale bar: $2 \mu \mathrm{m}$. 


\section{Discussion}

Overall, our protein localization studies suggest the presence of Int in an organelle adjacent to the nucleus, very likely the apicoplast. It may reach this organelle thanks to its bipartite leader peptide. Higher resolution studies such as electron microscopy would be needed to allow the exclusion of the possibility of a localization within the innermost algal-derived membrane of the organelle instead of the lumen (see Fig 2). In addition, the localization experiments of Int in Toxoplasma by Sheiner et al (2011) [26] have been reproduced and confirmed by Farhat and Hakimi (not shown). They observed that Int was co-localized with the apicoplast during different plastid segregation stages. Accordingly, it can be argued that Int may be intimately associated with DNA transactions, namely duplication of the plastid DNA, at least in $T$. gondii.

As a putative member of the Y-SSR family (conserved active site residues, see S1 Fig), Int could possibly play a role in apicoplast genome decatenation, or genome separation by hairpin resolution after rolling circle replication. Many of the replication machinery proteins in the apicoplast are known, but this is the first report of the presence of an enzyme with the function of a type I topoisomerase in the apicoplast. Nevertheless, it is noteworthy that Y-SSRs could fulfill the role of other enzymes such as DNA ligase or telomerase that may also be involved in the maintenance of apicoplast genome copy number. Both Pf-int and type I topoisomerase rely on the catalytic tyrosine present in the Y-SSRs for the cleavage of DNA and have significant similarities in sequence and structure $[34,35]$. Topoisomerases proceed with either one or two strand cleavage (Type I/II), whilst Y-SSR usually cleave only one strand. Moreover, Y-SSRs require a specific sequence or structure, whereas topoisomerases are non-sequence specific [36]. The identification of specific DNA targets has so far been tricky, at least for Pf-Int. The potential DNA targets identified by SELEX and binding experiments by Ghorbal et al. (2012) [17] did not clearly yield a specific sequence motif. In this aspect, Pf-int could be similar to topoisomerases, which recognize DNA topology rather than specific sequences. 
272 Our cellular fractionation experiments showed that Pf-Int is present in the nuclear (thus apicoplast-) associated fraction. Moreover, dividing cells, i.e. schizonts, also show Pf-int within each daughter parasite. Functioning as a topoisomerase would mean that Int could play a role in the actual machinery of the plastid DNA replication. Ligase and telomerase activities are usually needed just after replication in order to seal the endings of the newly synthesized DNA molecule. Knowing that the apicoplast genome is present in circular as well as in hairpin-closed linear form, generated by Dloop- or rolling circle replication [10], both types of functions would be needed in the apicoplast.

HUH nucleases perform similar functions in rolling circle replication initiation and termination (Chandler et al 2013). HUH recognises hairpin structures (or the ssDNA at the 3' or 5' ends of the hairpin) [37] described to be present within the arms and at the center of the apicoplast genome's IR. The possibility for Int to perform a HUH nuclease-like function is therefore also justified.

Additionally, we hypothesize that Pf-int could have a role in resolving genome concatemers that occur during rolling circle replication. If rolling circle replication had its origin in the centre region of the IR, this is where the resulting concatemer would have to be resolved in a site-specific manner. This scenario however, does not explain the existence of linear apicoplast DNA molecules. But such linear DNA could have their endings 5'-3'sealed by a telomerase-like enzyme similar to the hairpin telomere resolvase ResT in Borrelia [38]. No such enzyme has been described in the apicoplast as yet. HUH nucleases, have been reported to often have, apart from the HUH domain, 291 domains with other activities, such as helicase or primase activity (Chandler et al. 2013). To this 292 end, also a domain with telomerase function could be imagined. However, as similar as HUH nucleases and Y-SSRs may be, there are also striking differences: In contrast to Y-SSRs, which establish the phosphotyrosine at the 3' end of the DNA, liberating a 5' $\mathrm{OH}, \mathrm{HUH}$ establishes the phosphotyrosine at the 5' end, liberating the 3' end for further processing (priming replication and/ 
296 or termination of replication). While Y-SSRs do not need high energy factors, HUH needs divalent 297 metal ions for activity [37].

299 In this work, in line with the T. gondii integrase localization results of Sheiner et al. (2011) [26], we 300 confirmed the probable sub-cellular localization of Pf-int to the apicoplast. This integrase, highly 301 conserved among Apicomplexa, shown to be essential at least in P. falciparum [18], and localized 302 to the apicoplast, where it may have an important function during DNA replication, could be a 303 potential novel drug target against diseases caused by Apicomplexans. In the future, this study 304 could be supplemented by pulling down Int together with apicoplast DNA, or atomic resolution 305 Cryo-EM of Pf-Int bound to apicoplast, in order to confirm this enzyme's function and validity as a 306 drug target. 


\section{Acknowledgments}

Prof. Dr. Artur Scherf (Unité de Biologie des Interactions Hôte-Parasite, Institut Pasteur, Paris, F-

31175015 ) for kindly providing consumables and lab space to A. V. Berglar for IFA experiments.

Dr. Shuai Ding (Unité de Biologie des Interactions Hôte-Parasite, Institut Pasteur, Paris, F-75015 ;

Prof. A. Scherf's lab) for providing the P. falciparum TPT-HA parasite strain.

Dr. Dayana C. Farhat \& Dr. Mohamed-Ali Hakimi (Institute for Advanced Biosciences (IAB),

Team Host-Pathogen Interactions and Immunity to Infection, INSERM U1209, CNRS UMR5309, and for contributing to the discussion in the manuscript.

\section{Author approvals}

322 All authors have seen and approved the manuscript, and it hasn't been accepted or published 323 elsewhere.

\section{Competing interests}

326 I have read BioRxiv's policy and the authors of this manuscript have the following competing

327 interests: AVB declares a potential conflict of interest in terms of funding for the $\mathrm{PhD}$ thesis by

328 Merck KGaA (Direct: employment, stock ownership, grants, patents). This does not alter our

329 adherence to BioRxiv policies on sharing data and materials. On behalf of all other authors, the 330 corresponding author declares that there are no conflicts of interest. 
1. WHO (2020) World malaria report 2020: 20 years of global progress and challenges.

2. WHO (2015) Toxoplasmosis Fact Sheet.

3. Moore RB, Oborník M, Janouskovec J, et al (2008) A photosynthetic alveolate closely related to apicomplexan parasites. Nature 451:959-963

4. McFadden GI, Reith ME, Munholland J, Lang-Unnasch N (1996) Plastid in human parasites. Nature 381:482

5. Haider A, Gupta A, Vaish S, Kumar B, Charan M, Mir SS, Tanveer A, Sinha A, Habib S (2012) Housekeeping and other metabolic functions of the Plasmodium plastid. Current Science 102:749-756 apicoplast. Philosophical Transactions of the Royal Society 365:749-763

7. Ralph SA, van Dooren GG, Waller RF, Crawford MJ, Fraunholz MJ, Foth BJ, Tonkin CJ, Roos DS, McFadden GI (2004) Metabolic maps and functions of the Plasmodium falciparum apicoplast. Nature Reviews Microbiology 2:203-216

8. Jomaa H, Wiesner J, Sanderbrand S, et al (1999) Inhibitors of the nonmevalonate pathway of isoprenoid biosynthesis as antimalarial drugs. Science 285:1573-1576

9. Ralph SA, van Dooren GG, Waller RF, Crawford MJ, Fraunholz MJ, Foth BJ, Tonkin CJ, Roos DS, McFadden GI (2004) Tropical infectious diseases: metabolic maps and functions of the Plasmodium falciparum apicoplast. Nature Reviews Microbiology 2:203-16

11. Waller RF, Keeling PJ, Donald RG, Striepen B, Handman E, Lang-Unnasch N, 
target to the plastid in Toxoplasma gondii and Plasmodium falciparum. Proceedings of the National Academy of Sciences of the United States of America 95:12352-12357

12. Waller RF, Reed MB, Cowman AF, Mcfadden GI (2000) Protein trafficking to the plastid of Plasmodium falciparum is via the secretory pathway. The EMBO Journal 19:1794-1802

13. Tonkin CJ, Struck NS, Mullin KA, Stimmler LM, McFadden GI (2006) Evidence for Golgi-independent transport from the early secretory pathway to the plastid in malaria parasites. Molecular Microbiology 61:614-630

14. Richardson LGL, Paila YD, Siman SR, Chen Y, Smith MD, Schnell DJ (2014)

Targeting and assembly of components of the TOC protein import complex at the chloroplast outer envelope membrane. Frontiers in Plant Science 5:269

15. Ling Q, Jarvis P (2015) Functions of plastid protein import and the ubiquitinproteasome system in plastid development. Biochimica et Biophysica Acta (BBA) Bioenergetics 1847:939-948

16. Wilson RJ, Denny PW, Preiser PR, et al (1996) Complete gene map of the plastid-like DNA of the malaria parasite Plasmodium falciparum. Journal of Molecular Biology 261:155-172

17. Ghorbal M, Scheidig-Benatar C, Bouizem S, Thomas C, Paisley G, Faltermeier C, Liu M, Scherf A, Lopez-Rubio J-J, Gopaul DN (2012) Initial Characterization of the Pf-Int Recombinase from the MalariaParasite Plasmodium falciparum. PloS one 7:e46507

18. Zhang M, Wang C, Otto TD, et al (2018) Uncovering the essential genes of the human malaria parasite Plasmodium falciparum by saturation mutagenesis. Science 360:1-26

19. Grindley NDF, Whiteson KL, Rice P a (2006) Mechanisms of site-specific recombination. Annual Review of Biochemistry 75:567-605 
20. Reed RR, Grindley ND (1981) Transposon-mediated site-specific recombination in vitro: DNA cleavage and protein-DNA linkage at the recombination site. Cell 25:721728

21. Escudero JA, Loot C, Nivina A, Mazel D (2014) The Integron: Adaptation On Demand. Microbiology Spectrum 3:MDNA3-0019-2014

22. Cody JP, Graham ND, Zhao C, Swyers NC, Birchler JA (2020) Site-specific recombinase genome engineering toolkit in maize. Plant Direct 4:1-9

23. Craig NL (2015) Mobile DNA III, 3rd ed. IEEE Micro. https://doi.org/10.1109/MM.2009.63

24. Ghorbal M (2012) Caractérisation biochimique, structurale et fonctionnelle de Pf-Int : une recombinase à site spécifique potentielle de Plasmodium falciparum. Université Paris-Sud 11

25. Prasad A, Mastud P, Patankar S (2020) Dually localized proteins found in both the apicoplast and mitochondrion utilize the Golgi-dependent pathway for apicoplast targeting in Toxoplasma gondii. Biology of the Cell. https://doi.org/10.1111/boc.202000050 
29. Walliker D, Quakyi IA, Wellems TE, McCutchan TF, Szarfman A, London WT, Corcoran LM, Burkot TR, Carter R (1987) Genetic analysis of the human malaria parasite Plasmodium falciparum. Science 26:1661-1666

30. Trager W, Jensen JB (1967) Human Malaria Parasites in Continuous Culture. Science 193:673-675

31. Lambros C, Vanderberg JP (1979) Synchronization of Plasmodium falciparum Erythrocytic Stages in Culture. The Journal of Parasitology 65:418-420

32. Oehring SC, Woodcroft BJ, Moes S, et al (2012) Organellar proteomics reveals hundreds of novel nuclear proteins in the malaria parasite Plasmodium falciparum.

33. Mullin K a, Lim L, Ralph S a, Spurck TP, Handman E, McFadden GI (2006) Membrane transporters in the relict plastid of malaria parasites. Proceedings of the National Academy of Sciences of the United States of America 103:9572-9577

34. Perry K, Hwang Y, Bushman FD, Van Duyne GD (2010) Insights from the Structure of a Smallpox Virus Topoisomerase-DNA Transition State Mimic. Cell Press 18:127137

35. Gibb B, Gupta K, Ghosh K, Sharp R, Chen J, Van Duyne GD (2010) Requirements for catalysis in the Cre recombinase active site. Nucleic Acid Research 38:5817-32

36. Minkah N, Hwang Y, Perry K, Van Duyne GD, Hendrickson R, Lefkowitz EJ, Hannenhalli S, Bushman FD (2007) Variola virus topoisomerase: DNA cleavage specificity and distribution of sites in Poxvirus genomes. Virology 365:60-69 
bioRxiv preprint doi: https://doi.org/10.1101/2021.10.12.464051; this version posted October 12, 2021. The copyright holder for this preprint (which was not certified by peer review) is the author/funder. All rights reserved. No reuse allowed without permission.

432 